\title{
Tactile, maternal, and pharmacologic factors involved in the "transport response" in rat pups
}

\author{
CHRISTOPHER WILSON, ALLISON D. CROMEY, and ERIC KRAMER \\ Lafayette College, Easton, Pennsylvania
}

\begin{abstract}
A series of eight experiments was run to characterize the potentiation of the transport response reported with tactile stimulation. Experiments $1 \mathrm{~A}-\mathrm{C}$ were designed to investigate the types of tactile stimulation that could potentiate an initially weak transport response. Experiments $2 \mathrm{~A}-\mathrm{C}$ were designed to assess the effect of maternal/ittermate deprivation on responsiveness to external stimuli in 9- and 16-day-old rat pups. Experiments 3A and 3B were designed to investigate, pharmacologically, underlying mechanisms for the transport response potentiation effect. It was found that if the initial stimulus in producing a strong transport response is inadequate, additional tactile stimulation can potentiate the response. The additional stimuli work best if they are diffuse, if discrete inputs can summate over areas, or if they encompass the snout area. It also was found that isolation could lead to increased responsiveness to stimuli normally eliciting or potentiating the response in 9- and 16-day-old rats. Finally, results indicate that both dopaminergic and beta-noradrenergic systems are important in the response potentiation seen with maternal/littermate deprivation.
\end{abstract}

In nonprecocial animals, very specific behaviors seen in the infant must be coordinated with behaviors of the parent to ensure the infant's survival. Brewster and Leon (1980) described a behavior in infant rats that enables the mother to transport the infant efficiently from one location to another. This "transport response" is characterized by an active flexion and adduction of the hindlimbs, an extension and adduction of the forelimbs, and, in some cases, an adduction of the tail to the ventral surface of the body. The behavior, made in response to stimulation by the mother, is elicited most effectively when the mother grasps the pup's dorsal surface, including the skin of the nape of the neck (Brewster \& Leon, 1980). The response to this stimulus provides a relatively compact package for maternal transport at a time when the pup is fairly active and relatively unwieldly (Brewster \& Leon, 1980; Wilson, 1985; Wilson, Cullen, $\&$ Sendell, 1984) and, thus, is ecologically important.

In a recent paper, Wilson (1988) reported that external tactile stimulation in the form of an air puff, water puff, or tail pinch could potentiate a weak transport response. Stimuli such as these have been shown to induce goal-directed behaviors such as eating, gnawing, and licking (Antelman \& Szechtman, 1975) and have been purported to engage a central dopmine system (Antelman \& Szechtman, 1975; Camp \& Rudy, 1987). In fact, neurochemical systems mediating many forms of behavioral arousal appear to be largely catecholaminergic in nature. For instance, many authors have implicated dopamine involvement in general arousal (Campbell, Lytle, \& Fibiger, 1969; Fibiger, Lytle, \& Campbell, 1970) and in the

All correspondence concerning this article should be directed to Christopher Wilson, Department of Psychology, Sam Houston State University, Huntsville, TX 77341. production of various forms of stereotypy (Barrett, Caza, Spear, \& Spear, 1982; Costall \& Naylor, 1973; Ernst, 1967; Lal \& Sourkes, 1973; Melzacka, Wisniowska, \& Vetulani, 1978; Reinstein, McClearn, \& Isaacson, 1978).

Hall (1979a) reported that intraoral infusions of milk evoked increases in behavioral arousal in 3-day-old rat pups, as manifested in active head probing and rolling. If the pups were subjected to increasing levels of maternal deprivation, they tended to show increasing levels of arousal when given the milk stimulus. In a later paper, Hall (1979b) reported that maternal deprivation resulted in ontogenetic variations in arousal responses. He reported a much greater deprivation effect in 3- to 12-day-old pups than in 15- and 20-day-old pups. The lack of effect of deprivation on general activity in 15-day-old rats is in line with similar results reported by Hofer (1970), who reported that maternal deprivation in 14-day-old pups did not evoke changes in general activity. Descriptively, the arousal responses described by Hall $(1979 a, 1979 b)$ are diffuse and general, marked by rolling, curling, and wall climbing. As these animals mature, the responses to environmental stimulation become directed, focused, and stimulation-bound (Szechtman \& Hall, 1980).

General behavioral activation induced by milk infusions as well as more focused, discrete arousal responses appear to be mediated by dopamine systems (Antelman \& Szechtman, 1975; Camp \& Rudy, 1987). We have reported that the transport response is probably dopaminergically mediated, since haloperidol can reduce the intensity of the response in 19-day-old rat pups (Wilson et al., 1984) and apomorphine can reinstate the response in 32-, 34-, and 36-day-old rats, animals normally too old to show an effective transport response. As in other forms of behavioral arousal, the transport response also appears to be suppressed by later-developing cholinergic systems 
(Wilson \& Cromey, 1989). The transport response is not diffuse or general in nature, but rather is specific and requires that the animal assume and maintain a rather complex posture. Since the response occurs only after the first week of life, a period of time when the pups are making a transition from general, nondirected behaviors to more discrete, directed, responses, we believe that the transport response may be a member of the set of responses usually described under the category of arousal responses, à la Szechtman and Hall (1980).

This series of experiments was designed to characterize and categorize further the transport response and to investigate the mechanisms involved in the response potentiation seen with application of external stimuli. Experiments $1 \mathrm{~A}-\mathrm{C}$ were designed to elucidate further the attributes of the additional tactile stimulation that make it potentiate an initially weak transport response. The remaining experiments were designed to investigate whether the transport response might be considered, behaviorally and pharmacologically, an aspect of behavioral arousal. Experiment 2A was designed to determine whether isolation would lead to an increase in sensitivity to dorsal stimulation, stimulation that normally can induce a transport response. Experiments $2 \mathrm{~B}$ and $\mathrm{C}$ were designed to determine whether isolation would lead to an increase in sensitivity to stimuli that have been found to potentiate the transport response at various levels. Experiments 3A and $3 \mathrm{~B}$ were designed to investigate, pharmacologically, underlying neurotransmitter systems involved in changes in response potentiation seen with isolation.

\section{GENERAL METHOD}

\section{Subjects}

The subjects in these experiments consisted of 210 SpragueDawley albino rats, $9,15,16$, or 30 days of age at the time of testing. Litters were derived from breeding colonies in the Department of Psychology at Lafayette College. All litters were housed with their mothers in clear Plexiglas breeding cages in a room kept at $22^{\circ} \mathrm{C}$ on a 12:12-h light:dark cycle, with lights on at $0700 \mathrm{~h}$. All testing occurred between 1100 and $1200 \mathrm{~h}$.

\section{Apparatus}

In Experiments 2A-C, 3A, and 3B, we used an incubator designed roughly after that described by Hall $(1979 \mathrm{~b})$. The incubator was constructed out of a 10-gallon aquarium and contained an $80-\mathrm{mm}$ cooling fan (Archer, Cat. No. 273-242), capable of an air flow of $32 \mathrm{ft}^{3} / \mathrm{min}$, which blew over a 60 -W light bulb into a pan of water. The aquarium was fitted with a Plexiglas top, which was slightly ajar. This construction allowed for the maintenance of an internal temperature of $34^{\circ} \pm 1^{\circ} \mathrm{C}$ with between $40 \%$ and $50 \%$ humidity. The system was in operation during the entire time any pups were placed in the incubator.

\section{Materials}

Haloperidol generously was supplied by McNeil Pharmaceuticals and propranolol was purchased from Sigma Chemical Company.

\footnotetext{
Procedure

Approximately 4 days prior to parturition, pregnant female rats were placed in clear Plexiglas breeding chambers containing nest-
}

ing material. Each day, at 0900 and $1700 \mathrm{~h}$, the cages were checked for the presence of newborn litters. The first day a litter was present was recorded as postnatal Day 0 . On the afternoon of postnatal Day 1, each litter was culled to 8 to 10 pups; litters containing fewer than 8 pups were not used in these experiments.

In Experiments $2 \mathrm{~A}$ and 2B,8-day-old litters were split. Some of the pups in each litter were placed in the incubators $24 \mathrm{~h}$ prior to testing, some were placed in incubators $12 \mathrm{~h}$ prior to testing, and the remainder stayed with their mothers. Testing was carried out on Day 9; the pups in the incubators were removed and placed in individual styrofoam cups as were the pups that had remained with their mothers. In Experiment 2C, the litters were split on Day 15 exactly as in Experiments $2 \mathrm{~A}$ and $2 \mathrm{~B}$, with testing carried out on Day 16. In Experiments $3 A$ and 3B, the litters were split on Day 15, with some of the pups being placed in incubators $20 \mathrm{~h}$ prior to testing and the remainder of the litter staying with their mothers. Testing in these experiments occurred on Day 16.

At the time of testing, the rats were suspended by the skin of the nape of the neck with a spring-mechanized clothespin and suspended above the surface of a table. In Experiments $1 \mathrm{~A}-\mathrm{C}, 2 \mathrm{~B}-\mathrm{C}$, and $3 \mathrm{~A}-\mathrm{B}$, within $2 \mathrm{sec}$ after each pup was suspended, it was given the first of an individually predetermined, randomly ordered, sequence of tactile stimulus presentations. The stimulus presentations in these experiments included a no-stimulus control condition, a puff of water to the ventrum from an atomizer, or a jet(s) of water $(0.1 \mathrm{ml})$ to the middle of the chest delivered in more than $1 \mathrm{sec}$ from a 26-ga needle(s) attached to a 1.0-ml syringe. Immediately following a given presentation, the response was photographed for later scoring and the rat was placed back in its styrofoam cup. After all the pups in the litter had been given their first stimulus presentation, the procedure was repeated until each pup had received its entire sequence of stimulus presentations. (For a more detailed description of this procedure, see Wilson, 1988.) As a check for any gross motor impairments, at the conclusion of testing in each experiment, each rat pup was given a righting response test by being placed on its back: whether the animal righted itself within $1 \mathrm{sec}$ was recorded. Since all animals involved in all the experiments presented here were able to right themselves, this information is not presented for individual experiments.

\section{Response Scoring and Data Analysis}

Scoring of the responses followed the schema proposed by Brewster and Leon (1980). Each pup's response was graded on a scale of $0-5$, with 1 point being awarded for each forelimb, hindlimb, and/or tail that the pup brought into contact with its ventral surface. Data were analyzed using standard parametric procedures, with subsequent comparisons between conditions being determined with Newman-Keuls post hoc tests (Kirk, 1968). Differences with a probability of less than $.05(p<.05)$ were considered statistically reliable.

\section{EXPERIMENTS 1A-C}

Wilson (1988) has shown that the most effective types of stimuli in potentiating the transport response when the primary stimulus, a pinch to the nape of the neck, proves inadequate, were tactile in nature. Stimuli such as those employed by Wilson (1988) have been shown to induce various forms of behavioral arousal (Antelman \& Szechtman, 1975) and may engage dopaminergic systems (Camp \& Rudy, 1987). Experiments 1A-C were designed to better characterize the nature of the tactile stimulus in potentiating an initially weak transport response. 


\section{Experiment 1A}

Experiment $1 \mathrm{~A}$ was designed to determine whether the potentiating tactile stimulus needed to be widespread and diffuse, as in an air puff or water puff, or whether a single, well-defined stimulus could potentiate an initially weak transport response.

\section{Method}

Twenty-four 15-day-old rat pups were elevated and suspended from a spring-mechanized clothespin, such that each pup displayed, at best, only a very weak transport response. The pups were then subjected to a series of additional stimulus presentations, including no stimulus, the mist from an atomizer, or the squirt of water $(0.1 \mathrm{ml})$ from a $26-\mathrm{ga}$ needle attached to a $1.0-\mathrm{ml}$ syringe. The mist from the atomizer, delivered to each pup's ventral surface, encompassed the chest, abdomen, forelimbs, and hindlimbs. The squirt, delivered manually by the experimenter, was delivered in less than $1 \mathrm{sec}$ from a distance of $1 \mathrm{~cm}$ and was aimed at the pup's chest just at the point of the abdominal cavity and the sternum.

\section{Results}

Results for Experiment 1A are presented in the top panel of Figure 1. A repeated measures analysis of variance (ANOVA) on response intensity yielded a signifi-
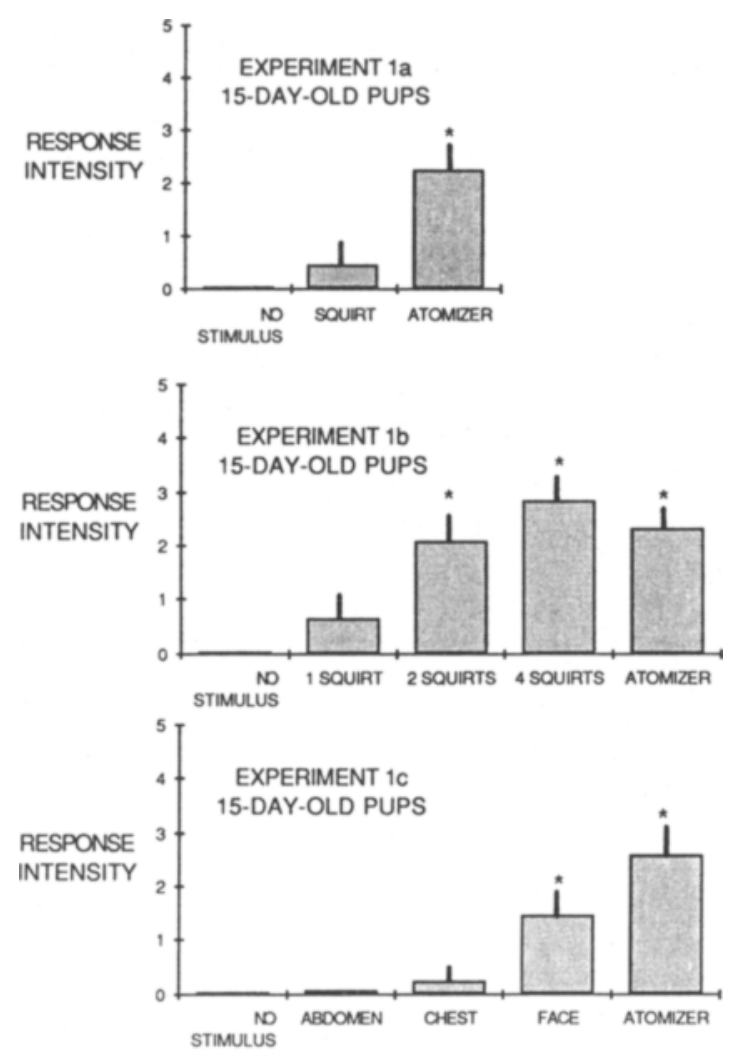

STIMULUS CONDITION

Figure 1. Transport response intensities $(+1 S E M)$ as a function of nature of stimulus condition in 15-day-old rats (* denotes reliably different from the no-stimulus control condition). cant trial effect $[F(2,46)=64.88, p<.05]$. NewmanKeuls post hoc tests revealed that the diffuse spray from the atomizer resulted in an increment in response intensity as compared with the no-stimulus situation and the discrete squirt condition. No other differences were statistically reliable.

\section{Experiment 1B}

This experiment was designed to determine whether increasing the number of the discrete water jets would increase transport response intensity.

\section{Method}

Sixteen 15-day-old pups were prepared as in Experiment $1 \mathrm{~A}$. In this experiment, though, the series of stimulus presentations included a no-stimulus situation, a squirt of water from one, two, or four 26-ga needles attached to $1.0-\mathrm{ml}$ syringes, or a spray of mist over the ventrum from an atomizer. When more than one squirt was used, the needles were arranged side by side (two squirts) or in a square (four squirts), with a distance of $1.5 \mathrm{~cm}$ separating the needles. Squirts were arranged such that the pups were stimulated on the lower abdominal and midchest regions (two squirts) and under the forelimbs and over the hindlimbs (four squirts). As before, each rat received the entire sequence, which was randomly ordered.

\section{Results}

Results for this experiment are presented in the middle panel of Figure 1. A repeated measures ANOVA on response intensity yielded a significant trial effect $[F(4,60)=$ $28.20, p<.05$ ]. Newman-Keuls post hoc analyses revealed a significant increase in transport response intensity over the no-stimulus condition when two or more needles or the atomizer was used as the supplemental stimulus.

\section{Experiment 1C}

Experiment 1C was designed to determine whether different body areas might be more susceptible to producing response potentiation when stimulated.

\section{Method}

Twenty-four 15-day-old pups were prepared as in Experiments 1A-B, except that the series of stimulus presentations included a no-stimulus condition, conditions of one squirt of water to the lower abdomen, one squirt to the chest above the sternum, one squirt to the side of the snout containing the vibrissae, or the delivery of a mist from an atomizer to the snout containing the vibrissae. When the snout area involved, the side stimulated was randomly chosen.

\section{Results}

Results for Experiment $1 \mathrm{C}$ are presented in the bottom panel of Figure 1. A repeated measures ANOVA on response intensity yielded a significant trial effect $[F(4,92)=$ $64.59, p<.05]$. Post hoc analyses revealed increments in response intensity over the control condition when a squirt to the snout area or the atomizer was used as a supplemental stimulus. Furthermore, the diffuse spray of the 
atomizer to the face produced an increment in response intensity above that of the squirt to the face alone. No other differences were statistically reliable.

\section{Discussion}

Data presented here replicate results reported by Wilson (1988) that additional tactile stimulation can potentiate or intensify the transport response if the initial stimulus, a pinch to the nape of the neck, is inadequate in producing a strong response. Data presented here indicate that the additional tactile stimuli work best if they are diffuse, if more than one input can be spatially summated, or if the stimulus involves the snout area.

\section{EXPERIMENTS 2A-C}

Pharmacologically, initiation of the transport response appears to be under dopaminergic control (Wilson, 1985; Wilson et al., 1984), whereas suppression of the response appears to be under cholinergic control (Wilson \& Cromey, 1989). Similar pharmacological effects have been reported with behavioral arousal (Camp \& Rudy, 1987; Campbell et al., 1969), and, thus, the transport response may be a member of the set of responses typically categorized as arousal responses. Hall (1979b) reported increases in response vigor elicited by arousal-producing stimuli following maternal deprivation, and this effect appears to be dopamine dependent (Camp \& Rudy, 1987). Experiments $2 \mathrm{~A}-\mathrm{C}$ were designed to determine whether maternal deprivation would result in increased responsiveness to stimuli that are known to induce a transport response or that have been reported to potentiate an initially weak response.

\section{Experiment 2A}

Experiment 2A was designed to determine whether maternal deprivation could lead to an increase in responsiveness to a dorsal pinch, a stimulus normally associated with the mother's grasping the infant for transport. Since the transport response first appears around Day 8 , we used 9-day-old pups in this experiment to see whether there would be an effect of deprivation on elicitation of the response.

\section{Method}

Three 8-day-old litters (24 pups) were split into 3 groups, as described in the General Method section, with some of the pups being placed in incubators and others returned to their mothers. The following day, 9-day-old pups that had been deprived of their mothers and littermates for varying periods of time $(0.25,12$, and $24 \mathrm{~h}$ ) were tested for the presence and intensity of the transport response. The pups were removed from the incubator or the home nest and placed in individual styrofoam cups. The testing procedure consisted of picking up each pup by the nape of the neck with a spring-mechanized clothespin and suspending it above the surface of a table. In this experiment, the pup was placed in the apparatus carefully but no care was taken to ensure that the pup did not assume a transport response caused by the pressure from the clothespin. After all the pups in the litter had been given their first trial, the procedure was repeated; the mean transport response in- tensity of the two trials served as the dependent variable in this experiment.

\section{Results}

Results for Experiment 2A are presented in Figure 2. The data show increasing levels of response intensity as a result of increasing levels of deprivation. An ANOVA performed on differences in mean response intensitities yielded a significant effect of deprivation $[F(2,21)=7.06$, $p<.05]$. Newman-Keuls post hoc tests revealed a reliable increase in transport response intensity for pups in both the 12-h and 24-h deprivation groups, as compared with pups in the 0.25 -h deprivation condition.

\section{Experiment 2B}

The results reported in Experiment 2A, like those reported by Hall (1979b), indicate that deprivation can lead to increases in responsiveness to environmental stimuli. This experiment was designed to determine whether maternal/littermate deprivation would lead to an increased sensitivity to additional tactile stimuli-stimuli that can produce a potentiation in the transport response (Wilson, 1988).

\section{Method}

In Experiment 2B, 24 9-day-old rat pups, which had been deprived of their mothers and littermates for varying periods of time $(0.25$, 12 , or $24 \mathrm{~h}$ ), were tested for the presence and intensity of the transport response in the manner described above for Experiment $2 \mathrm{~A}$. The pups were suspended individually by the skin of the nape of the neck with a clothespin. In this experiment, because we were interested in testing the abilities of different stimuli to potentiate the transport response, care was taken so that the pups would relax and not exhibit the classic transport response reported by Brewster and Leon (1980). At this point, each pup was tested with a sequence of tactile stimulus presentations as described above in the General Method section. The presentations again included a no-stimulus control condition, a puff of water to the ventrum from an atomizer, or a jet of water.

\section{Results}

Data for this experiment are presented in the top panel of Figure 3. The data show increases in responsiveness to potentiating stimuli with increases in deprivation times.

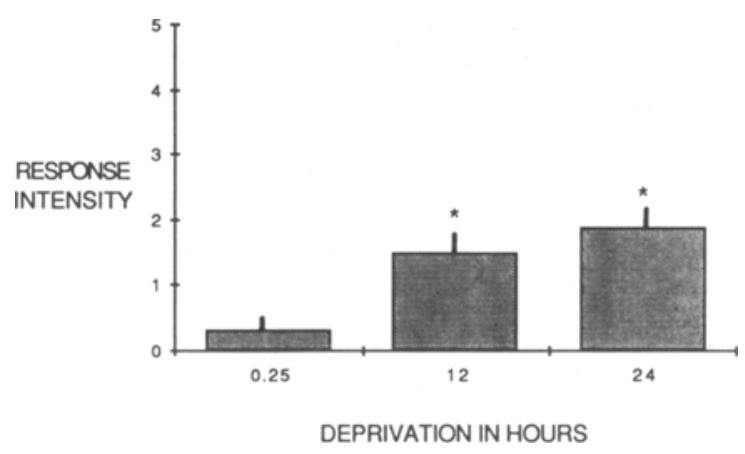

Figure 2. Transport response intensities $(+1 S E M)$ as a function of level of deprivation in 9-day-old rats $(*$ denotes reliably different from 0.25-h deprivation condition). 


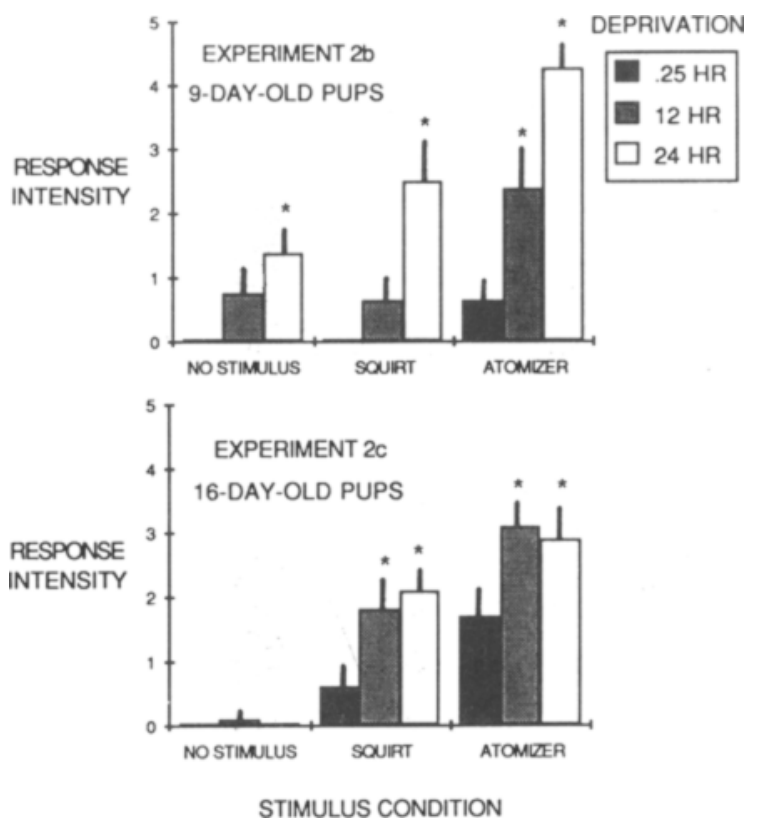

Figure 3. Transport response intensities $(+1$ SEM) as a function of nature of stimulus condition and level of deprivation in 9- and 16-day-old rats (* denotes reliably different from 0.25 -h deprivation condition for that stimulus condition).

An ANOVA performed on differences in mean response intensities yielded a significant deprivation effect $[F(2,21)=$ $23.32, p<.05]$, a significant stimulus condition effect $[F(2,42)=22.70, p<.05]$, and a significant deprivation $\times$ stimulus condition interaction $[F(4,42)=3.33$, $p<.05]$. Post hoc analyses revealed an increase in response intensity following $24 \mathrm{~h}$ of deprivation for pups in the no-stimulus condition. Post hoc analyses also revealed stronger levels of responding to the squirt in the 24-h deprived pups and to the atomizer in both the 12and 24- $h$ deprived subjects, as compared with the 0.25 - $h$ deprived rats in those respective conditions.

\section{Experiment 2C}

Experiment 2B showed a transport response potentiation with additional tactile stimulation in 9-day-old rat pups. This potentiation increased with maternal/littermate deprivation. While Hall (1979b) reported a lack of general behavioral arousal in 15-day-old rats, Szechtman and Hall (1980) reported increments in focused behavioral arousal as a result of tail pinches in 15- to 30-day-old rats. Experiment $2 \mathrm{C}$ was designed to investigate whether maternal/littermate deprivation would lead to an increase in transport response potentiation in 16-day-old rats. If the mechanisms involved in the forms of behavioral activation reported by Szechtman and Hall (1980) are the same as those of the transport response, we might expect an increase in transport response potentiation in the deprivation procedure.

\section{Method}

On postnatal Day 16, 30 rat pups that had been deprived of their mothers and littermates for varying periods of time $(0.25,12$, or $24 \mathrm{~h}$ ) were tested for the presence and intensity of the transport response as described in Experiment 2B.

\section{Results}

Results for Experiment $2 \mathrm{C}$ are presented in the bottom panel of Figure 3. The data show an increase in response potentiation with extended maternal deprivation in the water jet and atomizer conditions, with little or no differences in the no-stimulus control condition. An ANOVA on response intensities yielded a reliable deprivation effect $[F(2,27)=10.92, p<.05]$, a reliable stimulus effect $[F(2,54)=50.73, p<.05]$, and no deprivation $\times$ stimulus interaction $[F(4,54)=2.07, p>.05]$. Post hoc analyses revealed increases in the level of transport response potentiation in the squirt and atomizer conditions in pups subjected to 12 or $24 \mathrm{~h}$ of deprivation as compared with pups in the 0.25 -h group.

\section{Discussion}

The data from Experiments $2 \mathrm{~A}-\mathrm{C}$ appear fairly clear: Deprivation produces an increase in response intensity evoked by stimuli that have been shown to induce or potentiate the transport response, and this effect occurs in 9- and 16-day-old rats. Interestingly, in Experiment 2C, we recorded an increase in response potentiation with a squirt in the 12-h deprived rats, an effect not reported in Experiment 2B. The lack of effect in Experiment 2B may have been due to error variance, or it may indicate that the response potentiation had continued to develop, making the animal very responsive to tactile stimuli at 16 days of age. The data are consistent with the changes in focused activities with tail pinch reported by Szechtman and Hall (1980).

\section{EXPERIMENT 3A-B}

In rats under 8 days of age, arousal responses are general and very diffuse; after this age, these general, nondirected responses disappear, and behavioral arousal is reflected by more discrete responses (Camp \& Rudy, 1987). The transport response is a discrete response that can occur after administration of stimuli that result in behavioral arousal, and it does not occur until the second week of life (Brewster \& Leon, 1980). Experiments 1A-C and $2 \mathrm{~A}-\mathrm{C}$ revealed that tactile stimulation could potentiate the transport response and that maternal/littermate deprivation could cause an increase in responsiveness to those potentiating stimuli. Therefore, from a behavioral standpoint, we have hypothesized that the transport response may be representative of response activation.

Camp and Rudy (1987) reported that the behavioral arousal resulting from milk infusions appears to be dopaminergically mediated. Likewise, Antelman and Szechtman (1975) reported that the behavioral arousal 
resulting from a mild tail pinch in adult rats appears to be mediated by dopamine systems, as does the transport response (Wilson, 1985; Wilson et al., 1984). Recent results from our laboratory indicate that the transport response potentiation resulting from additional tactile stimulation may be subserved by a system or systems other than dopamine, since haloperidol administration appears to be ineffective in suppressing the potentiation by an air puff (Wilson, 1988). Experiments 3A and 3B were designed to investigate the role of catecholaminergic mechanisms on the potentiation of the transport response.

Hofer (1970) pointed out that extended maternal deprivation in rat pups tends to induce a state similar to hibernation, with decreases in respiration and heart rate. In the previous experiments reported here, pups in the 24-h deprivation groups tended to look a bit ragged, and that level of deprivation appeared to be fairly extreme. We were concerned that pups in the 24-h deprivation condition were perhaps showing a general malaise, so the pups in Experiments 3A-B were therefore mother/littermatedeprived for a maximum of $20 \mathrm{~h}$. Furthermore, since most of our previous work had been carried out with rat pups around the age of 15 or 16 days, we chose to use pups at approximately those ages.

\section{Experiment 3A}

This study was designed to investigate the effects of blocking the dopaminergic system on the transport response potentiation seen with tactile stimulation and maternal/ littermate deprivation.

\section{Method}

On postnatal Day 16, 36 rat pups, which had been deprived of their mothers and littermates for varying amounts of time $(0.5$ and $20 \mathrm{~h}$ ), were given s.c. administrations of either haloperidol ( $2.5 \mathrm{mg} / \mathrm{kg} / 5 \mathrm{ml})$, a potent dopaminergic antagonist (Siegel, Albers, Agranoff, \& Katzman, 1981), or a saline control solution. All the pups were then put in individual containers and placed in the incubator for a 30-min postadministration interval to allow time for the drug to bind to the target issue. At the end of the 30-min interval, the pups were retrieved from the incubator and placed in individual styrofoam cups. At this point each rat was suspended and tested as described in Experiment 2B.

\section{Results}

Results for Experiment 3A are presented in Figure 4. The data show that deprivation increased responsiveness to the squirt and the atomizer. There also appears to be a reduction in the response intensity as a result of administration of haloperidol in the 0.5-h deprived animals. A three-factor ANOVA analyzing differences in mean response intensities yielded a nonsignificant drug effect $[F(1,32)=2.31, p>.05]$, a significant deprivation effect $[F(1,32)=22.52, p<.05]$, and a significant stimulus condition effect $[F(2,64)=48.38, p<.05]$. The drug $\times$ deprivation interaction was not significant $[F(1,32)=.90, p>.05]$, nor was the drug $\times$ stimulus condition interaction $[F(2,64)=2.20, p>.05]$. The deprivation $\times$ stimulus interaction was reliable $[F(2,64)=3.85, p<.05]$, and the drug $\times$ deprivation

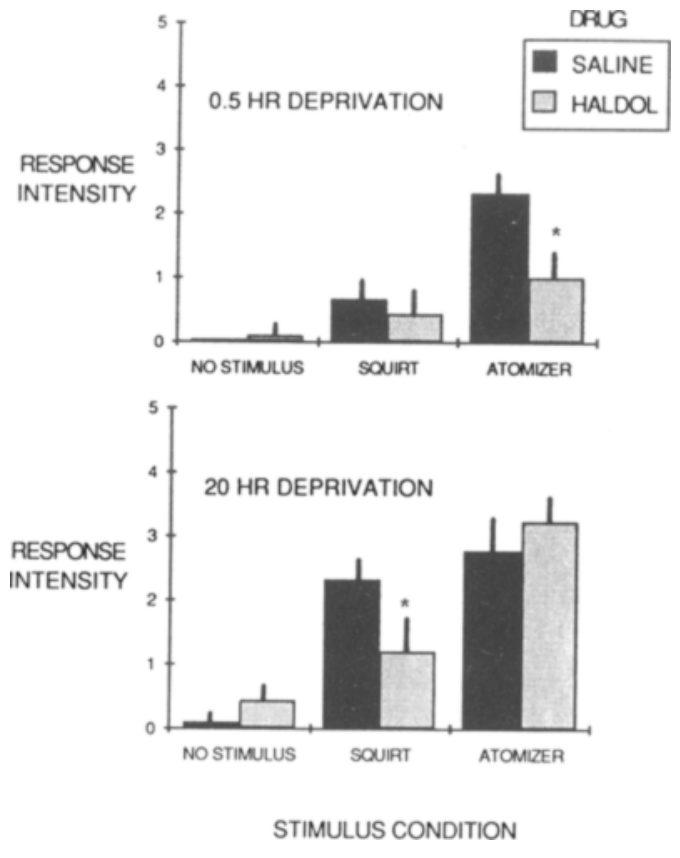

Figure 4. Transport response intensities $(+1 S E M)$ as a function of nature of stimulus condition, level of deprivation, and dose of haloperidol in 16-day-old rats (* denotes reliably different from saline group for that particular stimulus condition).

$\times$ stimulus condition interaction was also significant $[F(2,64)=4.04, p<.05]$. Post hoc analyses revealed that haloperidol produced an attenuation of the transport response in the 0.5 -h deprivation condition when the atomizer was used as the supplemental stimulus, but not when the squirt was used. Also, in the 20-h deprivation condition, haloperidol attenuated responding in the squirt condition, whereas this did not occur in the atomizer condition.

\section{Experiment 3B}

Experiment 3B was designed to investigate the effects of blocking the beta-noradrenergic system on the transport response potentiation seen with tactile stimulation and maternal/littermate deprivation.

\section{Method}

On postnatal Day 16, 32 rat pups, which had been deprived of their mothers and littermates for varying amounts of time $(0.5$ and $20 \mathrm{~h}$ ), were given s.c. administrations of either propranolol (20 $\mathrm{mg} / \mathrm{kg} / 5 \mathrm{ml}$ ), a classic beta-noradrenergic antagonist (Siegel, et al., 1981), or a saline control solution. The pups were then tested as described in Experiment 3A.

\section{Results}

Results for this experiment are presented in Figure 5. The data show that deprivation evoked increases in responsiveness to the squirt and to the mist from the atomizer, and that these increases may be blocked by propranolol. A three-factor ANOVA performed on differences in mean response intensities yielded a significant 

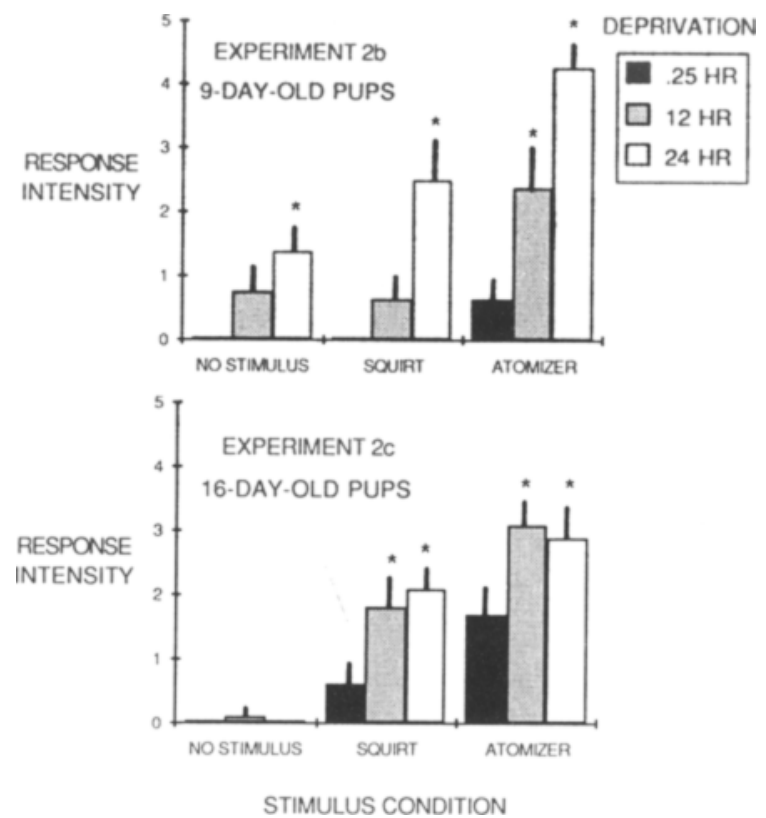

Figure 5. Transport response intensities $(+1 S E M)$ as a function of nature of stimulus condition, level of deprivation, and dose of propranolol in 16-day-old rats (* denotes reliably different from saline group for that particular stimulus condition).

drug effect $[F(1,28)=13.03, p<.05]$, a significant deprivation effect $[F(1,28)=20.12, p<.05]$, and a significant stimulus condition effect $[F(2,56)=56.34$, $p<.05]$. The drug $\times$ deprivation interaction effect was not significant $[F(1,28)=.97, p>.05]$, but the drug $X$ stimulus condition interaction was significant $[F(2,56)=$ $11.19, p<.05]$. The deprivation $\times$ stimulus condition interaction was reliable $[F(2,56)=3.20, p<.05]$, but the drug $\times$ deprivation $\times$ stimulus condition triple interaction was not reliable $[F(2,56)=1.96, p>.05]$. Post hoc analyses revealed that propranolol was able to suppress the response potentiation seen in the squirt condition at $20 \mathrm{~h}$ of deprivation and in the atomizer condition regardless of deprivation level.

\section{Discussion}

Data from Experiments 3A and 3B indicate that perhaps more than one neurochemical system is involved in potentiating the transport response. We interpret the data from Experiment $3 \mathrm{~A}$ to indicate that dopamine system(s) may be involved in the potentiation of the transport response under normal circumstances. We did not get a reliable suppression of the transport response in the squirt condition with $0.5 \mathrm{~h}$ of deprivation. The reason for this lack of effect is, perhaps, that we are observing a "floor effect." The squirt produced no reliable increase in responding above the no-stimulus condition; if more than one system is involved in the response potentiation, then it might be very difficult to get a significant reduction in the response with such a small range with which to begin. The data from Experiment 3B indicate involvement of betanoradrenergic system(s) in the potentiation of the transport response with additional tactile stimulation in some situations. Perhaps it is the case that under certain circumstances, such as maternal deprivation, for example, more than one system is engaged by external stimulation to help promote retrieval by the mother.

\section{GENERAL DISCUSSION}

Experiments $1 \mathrm{~A}-\mathrm{C}$ replicate the previous finding of Wilson (1988) that additional tactile stimulation can potentiate or intensify the transport response if the initial stimulus is inadequate in producing a strong response. These data indicate that additional tactile stimuli work best in potentiating the response if they are diffuse, if more than one input can be spatially summated, or if the stimulus involves the snout area. The nature of these stimuli appears to be similar to that which can result in behavioral activation. From an ecological viewpoint, these results may indicate that the pup might intensify its response to maternal transport stimulation if it happens to be dragged by its mother.

Experiments $2 \mathrm{~A}-\mathrm{C}$ provide evidence that different levels of maternal/littermate deprivation can produce increases in pups' responsiveness to a pinch on the nape of the neck or to stimuli that can potentiate the transport response. These results were similar to those reported by Hall (1979b), who found increases in stimulation-bound activity with increases in maternal deprivation. Hall (1979b) reported increases in general activity in rats as young as 1,3 , and 6 days of age, a period in ontogeny too early to produce a transport response (Brewster \& Leon, 1980). Hall (1979b) also reported a lack of increased activity with deprivation by 15 days of age, but results from Experiment $2 \mathrm{~B}$ in the present study show a very strong effect of deprivation in pups at about this age. Hall (1979a, 1979b) was measuring generalized responsesrolling, curling - which disappeared by 15 days of age but were replaced by more discrete responses-licking, grooming, and probing. This latter set of responses may be related to those described by Antelman and Szechtman (1975) and Szechtman and Hall (1980), who reported licking, gnawing, and eating following tail pinch in developing rats.

Camp and Rudy (1987) reported results which indicate that many forms of behavioral activation are dopaminergically mediated. Previous work indicated that the emergence of this species-typical response is under control of a dopamine system(s) (Wilson, 1985; Wilson et al., 1984) and that the suppression of the response in the normal course of development is dependent upon development of central cholinergic systems (Wilson \& Cromey, 1989). Wilson and Cromey (1989) also suggested that the transport response seen in preweanling rat pups may be categorized under the rubric behavioral activation.

A recent report has suggested that demonstration of the transport response under some circumstances may be 
under control of neurochemical systems other than the dopamine system(s) (Wilson, 1988). Results from Experiment $3 \mathrm{~A}$ reported here add credence to that notion. Although haloperidol was effective in attenuating the transport response increment with the atomizer mist in the 0.5 - $\mathrm{h}$ deprivation condition, this dopaminergic blocker was ineffective in attenuating the response increment with this stimulus in the 20-h deprivation group. We also reported that haloperidol can block the increment in response intensity with maternal/littermate deprivation if the eliciting stimulus is fairly discrete, as in the water jet condition. These data lead us to the conclusion that, with little or no maternal/littermate deprivation or with a defined, discrete stimulus and 20-h deprivation, the mechanisms subserving the response attenuation are essentially the same as those subserving effects reported by Antelman and Szechtman (1975) and Camp and Rudy (1987). With $20 \mathrm{~h}$ of deprivation and a relatively diffuse tactile stimulus, systems other than dopamine may subserve the response elicitation. Under these circumstances, it may be that secondary or back-up systems are brought into play to cause the pup to respond in a particular way. This notion of back-up systems involved in the response elicitation was presented in a previous paper (Wilson, 1988). It was suggested that these systems could be induced when the primary mechanism is not fully developed or fully functional.

In Experiment 3B, we tested the involvement of betanoradrenergic systems in the transport response potentiation with deprivation combined with additional tactile stimulation. Results from this experiment show that the beta-noradrenergic blocker, propranolol, was able to suppress elicitation of the transport response effectively with both the squirt and mist from the atomizer in rats that had undergone $20 \mathrm{~h}$ of deprivation. In a previous paper, Wilson et al. (1984) reported a dose-dependent suppression of the transport response with propranolol in 19-dayold rat pups. That report, along with results reported here, point to beta-noradrenergic involvement in elicitation of the transport response. Wilson (1985) reported that administration of the beta-noradrenergic agonist isoproterenol is ineffective in reinstating the transport response in 32-, 36-, and 40-day-old rats, animals in which apomorphine was very effective in reinstating the response. That propranolol was effective in suppressing the increment in response intensity following deprivation suggests that the beta-noradrenergic systems may serve as a system for elicitation of the transport response in particular situations, perhaps as a backup, or secondary system. Regardless, taken all together, these reports suggest that the betanoradrenergic system serves in conjunction with the dopaminergic system to produce the transport response in different situations.

On the basis of the results reported here, we suggest that the transport response, a response that depends upon the active emitting and maintenance of a complex motor pattern, may be described within the category of "discrete arousal responses," à la Szechtman and Hall (1980). Thus, we suggest that the general behavioral arousal, evi- dent in rats during the first week or so, in the process of development, may become channeled into the transport response. The suppression of the response at about 28 to 30 days of age may be the result of further development of other neurotransmitter system(s).

\section{REFERENCES}

Antelman, S. M., \& Szechtman, H. (1975). Tail pinch induces eating in sated rats which appear to depend on nigrostriatal dopamine. Science, 189, 731-733.

Barrett, B. A., Caza, P., Spear, N. E., \& Spear, L. P. (1982). Wall climbing, odors for the home nest and catecholaminergic activity in rat pups. Physiology \& Behavior, 29, 501-507.

Brewster, J., \& LEON, M. (1980). Facilitation of maternal transport by norway rat pups. Journal of Comparative \& Physiological Psychology, 94, 80-88.

CAMP, L. L., \& RuDY, J. W. (1987). Behavioral activation in infant rats: Pharmacological evidence for dopaminergic mediation. Psychobiology, 15, 317-328.

Campbell, B. A., Lytle, L. D., \& Fibiger, H. C. (1969). Ontogeny of adrenergic arousal and cholinergic inhibitory mechanisms in the rat. Science, 166, 637-638.

Costall, B., \& Naylor, R. J. (1973). The role of telencephalic dopaminergic systems in the mediation of apomorphine-stereotyped behavior. European Journal of Pharmacology, 24, 8-24.

ERNST, A. M. (1967). Mode of action of apomorphine and dexamphetamine on gnawing compulsion in rats. Psychopharmacologia, 10, 316-323.

Fibiger, H. C., Lytle, L. D., \& Campbell, B. A. (1970). Cholinergic modulation of adrenergic arousal in the developing rat. Journal of Comparative \& Physiological Psychology, 72, 384-389.

HaLL, W. G. (1979a). Feeding and behavioral activation in infant rats. Science, 205, 206-209.

HaLL, W. G. (1979b). The ontogeny of feeding in rats: I. Ingestive and behavioral responses to oral infusions. Journal of Comparative \& Physiological Psychology, 93, 977-1000.

Hofer, M. A. (1970). Physiological responses of infant rats to separation from their mothers. Science, 168, 871-873.

KIRK, R. E. (1968). Experimental design: Procedures for the behavioral sciences. Belmont, CA: Brooks/Cole.

LAL, S., \& SoURKES, T. L. (1973). Ontogeny of stereotyped behavior induced by apomorphine and amphetamine in the rat. Archives of International Pharmacodynamics \& Therapeutics, 202, 171-182.

Melzacka, M., Wisniowska, G., \& Vetulani, J. (1978). The distribution of apomorphine in rat brain: Possible behavioral correlates. Polish Journal of Pharmacology, 30, 335-345.

Reinstein, D. K., McClearn, D., \& IsaAcson, R. L. (1978). The development of responsiveness to dopamine agonists. Brain Research, 150, 216-223.

Siegel, G. L., Albers, R. W., Agranoff, B. W., \& Katzman, R. (1981). Basic neurochemistry (3rd ed.). Boston: Little, Brown.

Szechtman, H., \& HaLL, W. G. (1980). Ontogeny of oral behavior induced by tail pinch and electrical stimulation of the tail in rats. Journal of Comparative \& Physiological Psychology, 94, 436-445.

Wilson, C. (1985). The effects of apomorphine and isoproterenol on the 'transport response' in white rats. International Joumal of Developmental Neuroscience, 3, 279-284.

WILSON, C. (1988). The effects of sensory stimulation in inducing or intensifying the "transport response" in white rats. Animal Learning \& Behavior, 16, 83-88.

Wilson, C., CROMEY, A. D. (1989). Evidence of a cholinergic input into the suppression of the transport response in white rats. Psychobiology, 17, 43-48.

Wilson, C., Cullen, E., \& Sendell, K. (1984). A pharmacologic investigation of the 'transport response' in the white rat. International Journal of Developmental Neuroscience, 2, 323-329.

(Manuscript received August 18, 1988; revision accepted for publication November 30,1988 .) 